\title{
Transitional cell carcinoma of the ovary (Review)
}

\author{
SATOSHI ICHIGO ${ }^{1}$, HIROSHI TAKAGI ${ }^{1}$, KAZUTOSHI MATSUNAMI ${ }^{1}$, \\ TAKAYUKI MURASE ${ }^{2}$, TSUNEKO IKEDA ${ }^{2}$ and ATSUSHI IMAI ${ }^{1}$ \\ Departments of ${ }^{1}$ Obstetrics and Gynecology, and ${ }^{2}$ Pathology, Matsunami General Hospital, Gifu 501-6062, Japan
}

Received June 21, 2011; Accepted October 12, 2011

DOI: $10.3892 / \mathrm{ol} .2011 .453$

\begin{abstract}
Transitional cell carcinoma (TCC) of the ovary is a rare recently recognized subtype of ovarian epithelial cancer. Ovarian TCC has a modest response to chemotherapy, and metastatic TCC from the renal pelvis results in mortality. The clinical presentation is indistinguishable from other types of ovarian carcinoma. Histopathological examination remains the first tool used in the diagnosis of these heterogeneous tumors and in the separation of closely related tumors. Since it is generally accepted that surgical resection is the primary therapeutic approach, and patient outcomes following chemotherapy are better than for other types of ovarian cancers, it is a reasonable concept to detect tumors when they are still confined within the ovaries. Thus, the aim of this review was to describe typical cases of primary TCC, and to review the medical literature for information on TCC management in order to determine appropriate diagnostic methods and therapy.
\end{abstract}

\section{Contents}

1. Introduction

2. General considerations

3. Incidence

4. Diagnosis

5. Treatment

6. Prognosis

7. Metastatic TCC to the ovary

8. Conclusion

\section{Introduction}

Ovarian cancer is the most lethal gynecological malignancy. Efforts at early detection and new therapeutic approaches to reduce mortality have largely been unsuccessful, since the

\footnotetext{
Correspondence to: Dr Atsushi Imai, Institute of Endocrine-Related Cancer, Matsunami General Hospital, Kasamatsu, Gifu 501-6062, Japan

E-mail: aimai@matsunami-hsp.org.jp
}

Key words: transitional cell carcinoma, paclitaxel, staging surgery, ovary, metastatic ovarian cancer origin and pathogenesis of epithelial ovarian cancer are poorly understood (1). Transitional cell carcinoma (TCC), a recently recognized subtype, resembles urothelium rather than ovarian surface epithelium (methothelium) (2,3). A small percentage of ovarian cancer types are accounted for by TCC, which has proven to be a distinct group with various histological and immunohistological patterns. Patients with TCC had better prognoses compared to patients with all other types of ovarian carcinomas following standardized chemotherapy (4). The aim of this review was to describe our typical cases of primary TCC, and to review the medical literature for information on TCC management in order to determine appropriate diagnostic methods and therapy.

\section{General considerations}

TCC of the ovary is a recently recognized subtype of ovarian surface epithelial cancer. TCC has been described as a primary ovarian carcinoma in which definite urothelial features are present, but no benign, metaplastic and/or proliferating Brenner tumor can be identified. TCC of the ovary was initally defined by Austin and Norris (5). These investigators reported a group of patients who had ovarian tumors presenting with histologic features similar to those observed in a malignant Brenner tumor, but the tumors lacked the associated benign Brenner tumor component. Pure TCC was thus distinguished from malignant Brenner tumor. In addition to not having a benign Brenner tumor component, TCC lacks the prominent stromal calcification (5-7). Since TCC of the ovary has close morphological similarities to TCC of the bladder and it behaves more aggressively than malignant Brenner tumors, Austin and Norris (5) concluded that ovarian TCC arises directly from the pluripotent surface epithelium of the ovary and from cells with urothelial potential, rather than from a benign or proliferative Brenner tumor precursor.

\section{Incidence}

The true incidence of TCC of the ovary remains unknown. Transitional cell tumors, including TCC, and benign and malignant Brenner tumors of the ovary represent $\sim 2 \%$ of all ovarian tumors. Moreover, according to the World Heath Organization (WHO), depending on the histological pattern, these tumors are classified as benign, borderline or malignant Brenner tumors and TCC (8). Silva et al (9) observed the focal or diffuse TCC pattern in 88 of 934 ovarian cancers $(9 \%)$. 
A

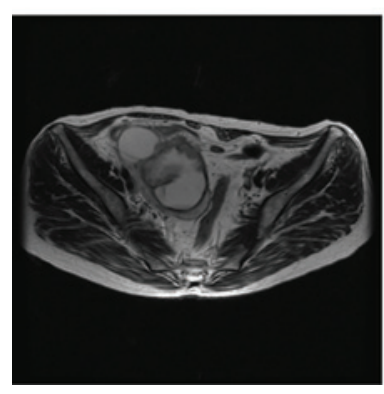

B

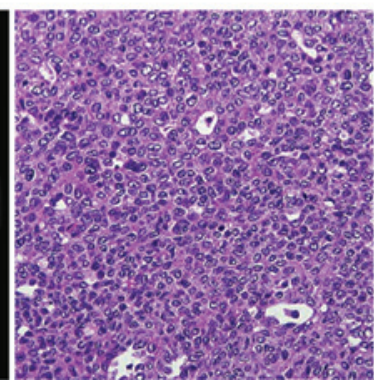

C

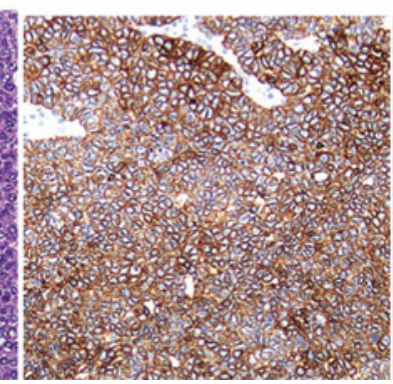

D

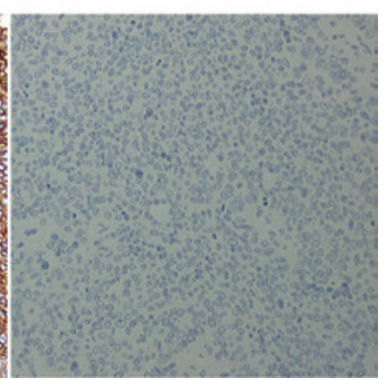

Figure 1. Primary TCC of the ovary (case 1). A 64-year-old postmenopausal woman presented with a 1-year history of progressive enlargement of an abdominal mass. Physical examination showed a pelvic mass. Abdominal ultrasound showed a pelvic mass measuring 6 cm with inhomogeneous echogenicity. (A) Horizontal T2-weighted MRI showed an inhomogeneous cyst on the right side of the pelvis, which was $>70$ mm in maximal diameter with a solid component. There was no evidence of lymphadenopathy. Prior to surgery, CA125 was elevated to $347 \mathrm{U} / \mathrm{ml}$ (normal, <35 U/ml), but other markers were all within normal ranges. Surgical staging procedures including total abdominal hysterectomy, bilateral salpingo-oophorectomy, infracolic omentectomy and pelvic lymph node dissection were performed. The ascites were also sent for cytological examination. (B) Microscopic examination showed a malignant transitional epithelial lining of the right ovarian cyst. The final diagnosis was TCC, grade 3, FIGO stage IIc. Immunohistochemical studies showed that the tumor was (C) positive for CK 7 and (D) negative for CK20. The patient received six cycles of chemotherapy with paclitaxel-carboplatin following surgery, and has been disease-free for 1 year.

A

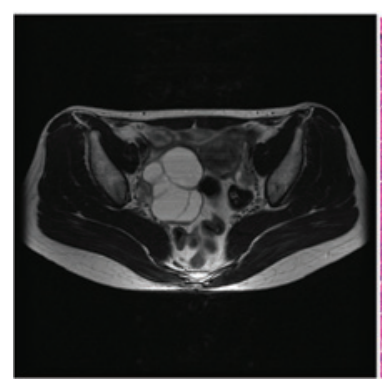

B

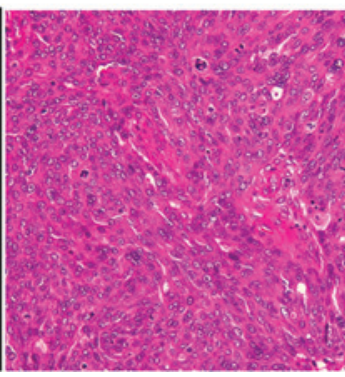

C

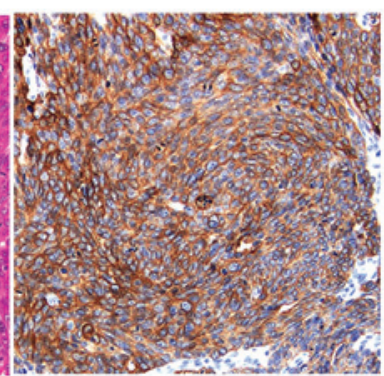

D

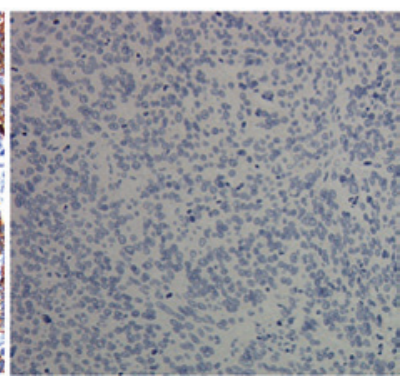

Figure 2. Primary TCC of the ovary (case 2). A 44-year-old woman with a right ovarian cyst was referred to the gynecology department. (A) The MRI (T2-weighted, horizontal) revealed a $5 \times 6 \mathrm{~cm}$ multiple cystic mass in the right adenexa, suggestive of an ovarian tumor. There was no obvious metastatic foci in other organs. The levels of serum CA72-4 and CA125 were $7.7 \mathrm{U} / \mathrm{ml}$ (normal, <10.0 U/ml) and 12.9 U/ml (normal, <10 U/ml), respectively. (B) The right ovarian cyst was laparoscopically resected and diagnosed as TCC, grade 3 with serosal involvement. (C) Immunohistochemical stains for CK7 were positive; (D) but staining for CK20 was negative. The ascites cytology was reported to be positive. The patient was staged as FIGO stage Ic. One month following adenectomy, the patient was submitted for exploratory staging procedures including total abdominal hysterectomy, bilateral salpingooophorectomy, infracolic omentectomy and pelvic lymph node dissection. The extensively sampled adnexal tissue was histologically uninvolved by the tumor. After recovering from surgery, the patient received six cycles of chemotherapy with paclitaxel-carboplatin. The patient is presently doing well without any recurrent disease for 2 years.

\section{Diagnosis}

The common presenting symptoms of TCC of the ovary are abdominal pain, abdominal swelling or distension and weight loss. Occasionally, the patient may present with uterine bleeding, back pain, bowel or urinary symptoms, as shown in our cases (Figs. 1 and 2). However, the clinical presentation is indistinguishable from other types of ovarian carcinoma $(5,10)$. As described in detail by Eichhorn and Young (10), ovarian TCC typically shows undulating, diffuse, insular and trabecular growth patterns. The tumor cell nuclei were oblong or round, often exhibiting nucleoli with longitudinal grooves. The cytoplasm was often pale and granular, and was rarely clear or eosinophilic (Figs. 1 and 2).

CA125 is clinically useful as a serum marker of tumor progression and recurrence, although early stages may be CA125-negative (Fig. 2). In the study by Ceauşu et al (11),
13 archived formalin-fixed paraffin-embedded samples of transitional cell tumors of the ovary were assessed using standard hematoxylin-eosin staining and the indirect tristadial ABC peroxidase immunohistochemistry method for 11 antibodies including CA125, cytokeratin (CK) 7 and CEA. Over $50 \%$ of the samples were malignant Brenner tumors, CA125 was positive in all malignant tumors (of Brenner type and TCCs), but not in the benign and borderline tumors, while CK7 was positive in approximately $70 \%$ of all cases. The two antibodies have shown a high sensitivity and low specificity, but do not correlate with each other. Recent findings $(11,12)$ have shown that p63 is expressed in benign and borderline Brenner tumors, but not in malignant counterparts and TCCs of the ovary, suggesting that this antigen is a marker for the differential diagnosis of malignant Brenner tumors and TCCs, and may also play a role in Brenner carcinogenesis. The aim of these studies was to detect tumors when they are still 
confined to the ovaries, thereby increasing the likelihood of cure and reducing the mortality of the disease. The modalities that are currently in use to screen women are pelvic examination, imaging modality and measurement of serum CA125 (1) (Figs. 1 and 2), although case 2 was CA125-negative.

\section{Treatment}

Optimal surgical resectability followed by cisplatin-based chemotherapy may contribute to the survival benefit $(4,6,7)$. The estimated 5-year survival following surgery for 88 patients was $37 \%$, whereas for patients who received chemotherapy, survival was at $41 \%(6,7)$. Factors associated with survival for patients who received chemotherapy were the clinical stage, the percentage of the TCC component in the primary tumor and the results of second-look surgery. The predominance of TCC was a favorable prognostic factor and patients with higher clinical stages had poorer prognoses.

\section{Prognosis}

The relative effects of tumor biology and treatment strategies remain undetermined $(6,7)$. Gershenson et al $(13,14)$ concluded that advanced-stage ovarian TCC was significantly more chemosensitive and associated with better prognosis than poorly differentiated serous carcinoma. Kommoss et al (4) also documented that patients with TCC had better prognoses compared to patients with all other types of ovarian carcinomas following standardized chemotherapy. The metastatic pathways of the tumor simulate TCC of the bladder, which implicates a loss of the integrity of E-cadherin (5-7).

\section{Metastatic TCC to the ovaries}

The ovaries are common sites for intra-abdominal metastasis (15). Approximately $6 \%$ of ovarian cancers found at laparotomy are secondary tumors from other sites $(15,16)$. Metastatic TCC from the urinary bladder, or elsewhere within the urinary system, involving the ovary is extremely rare (16). There have been six cases reported thus far, as described by Lee et al (17). In all cases, secondary ovarian tumors are unilateral. The time interval to the appearance of ovarian metastases varied from synchronous to 4 years. In the study by Lee $e t$ al (17), all cases received surgery, with the overall survival ranging from 3 months to 7 years. These cases favor metastatic ovarian tumors for the following reasons: definite histological evidence of a primary renal tumor, and deep stromal invasion. The origin of primary lesions has prognostic significance as TCC of the ovary has a modest response to chemotherapy (10) and metastatic TCC from the renal pelvis results in mortality (17).

Microscopically, metastatic TCC of the ovary resembles a primary ovarian TCC. Primary TCC accounts for 1-2\% of all ovarian tumors $(10,17)$. TCC of the ovary is a recently recognized subtype of ovarian surface epithelial-stromal cancer, and studies of its morphology are rare. The presence of a component of benign or borderline Brenner tumor confirms an ovarian primary tumor. TCC of the ovaries has mucin pools and thick papillae with smooth luminal borders, in contrast to the pseudo-papillae of tumor cell necrosis that is common in metastatic TCC $(10,17)$. Another study has shown that the morphological similarity between transitional cell carcinoma of the ovary and its counterpart from the urinary bladder does not indicate any histogenic similarity, but CK7 and CK20, together with uroplakin III and WT1 may prove useful in distinguishing primitive TCCs of the ovary, and metastases from invasive TCC of the bladder to the ovary, the former being a variant morphology in the spectrum of surface epithelial carcinomas $(11,18)$.

\section{Conclusion}

Microscopic examination remains the first tool in the diagnosis of these heterogeneous tumors and in the separation of closely related tumors. Primary TCC of the ovary is a relatively rare subtype of epithelial ovarian cancer. Surgical resection is the primary therapeutic approach, and patient outcomes following chemotherapy are better than for other types of ovarian cancers.

\section{References}

1. Kurman R and Shih I-M: The origin and pathogenesis of epithelial ovarian cancer: A proposed unifying theory. Am J Surg Pathol 34: 433-443, 2010.

2. Cuatrecasas M, Catasus L, Palacios J and Prat J: Transitional cell tumors of the ovary: A comparative clinicopathologic, immunohistochemical, and molecular genetic analysis of Brenner tumors and transitional cell carcinomas. Am J Surg Pathol 33: 556-567, 2009.

3. Oh S, Rha S, Jung S, Lee Y, Choi B, Byun J, Ku Y and Jung C: Transitional cell tumor of the ovary: computed tomographic and magnetic resonance imaging features with pathological correlation. J Comput Assist Tomogr 33: 106-112, 2009.

4. Kommoss F, Kommoss S, Schmidt D, Trunk M, Pfisterer J and du Bois A: Survival benefit for patients with advancedstage transitional cell carcinomas vs. other subtypes of ovarian carcinoma after chemotherapy with platinum and paclitaxel. Gynecol Oncol 97: 195-199, 2005.

5. Austin $\mathrm{R}$ and Norris $\mathrm{H}$ : Malignant Brenner tumor and transitional cell carcinoma of the ovary: A comparison. Int J Gynecol Pathol 6: 29-39, 1987.

6. Tazi E, Lalya I, Tazi M, Ahellal Y, M'rabti H and Errihani H: Transitional cell carcinoma of the ovary: a rare case and review of literature. World J Surg Oncol 8: 98-101, 2010.

7. Lin C, Liu F and Ho E: Transitional cell carcinoma of the ovary. Taiwan J Obstet Gynecol 45: 268-271, 2006.

8. World Health Organization: Classification of tumours: Pathology and genetics of tumors of the breast and female genital organs. IARC Press, Lyon, pp140-143, 2003.

9. Silva E, Robey-Cafferty S, Smith T and Gershenson D: Ovarian carcinomas with transitional cell carcinoma pattern. Am J Clin Pathol 93: 457-465, 1990.

10. Eichhorn J and Young R: Transitional cell carcinoma of the ovary: a morphologic study of 100 cases with emphasis on differential diagnosis. Am J Surg Pathol 28: 453-463, 2004.

11. Ceauşu M, Terzea D, Georgescu A, Dobrea C, Mihai M, Iosif C, Vasilescu F and Ardeleanu C: Transitional cell tumors of the ovary: a compact group with a heterogeneous histological and immunophenotypical pattern. Rom J Morphol Embryol 49: 513-516, 2008.

12. Liao X, Xue W, Shen D, Ngan H, Siu M and Cheung A: P63 expression in ovarian tumours: a marker for Brenner tumours but not transitional cell carcinomas. Histopathology 51: 477-783, 2007.

13. Gershenson D, Morris M, Burke T, Levenback C, Kavanagh J, Fromm G, Silva E, Warner D and Wharton J: Combined cisplatin and carboplatin chemotherapy for treatment of advanced epithelial ovarian cancer. Gynecol Oncol 58: 349-355, 1995.

14. Gershenson D, Silva E, Mitchell M, Atkinson E and Wharton J: Transitional cell carcinoma of the ovary: a matched control study of advanced-stage patients treated with cisplatin-based chemotherapy. Am J Obstet Gynecol 168: 1178-1185, 1993. 
15. Valappil S, Toon P and Anandaram P: Ovarian metastasis from primary renal cell carcinoma: report of a case and review of iterature. Gynecol Oncol 94: 846-849, 2004.

16. Skírnisdóttir I, Garmo $\mathrm{H}$ and Holmberg L: Non-genital tract metastases to the ovaries presented as ovarian tumors in Sweden 1990-2003: occurrence, origin and survival compared to ovarian cancer. Gynecol Oncol 105: 166-171, 2007.

17. Lee M, Jung Y, Kim S, Kim S and Kim Y: Metastasis to the ovaries from transitional cell carcinoma of the bladder and renal pelvis: a report of two cases. J Gynecol Oncol 21: 59-61, 2010.
18. Logani S, Olova E, Amin M, Folpe A, Cohen C and Young R: Immunoprofile of ovarian tumors with putative transitional cell (urothelial) differentiation using novel urothelial markers: histogenetic and diagnostic implications. Am J Surg Pathol 27: 1434-1441, 2003. 